\title{
Clinical profile of patients with polycystic ovarian syndrome in Nepal
}

\begin{abstract}
Background: Polycystic Ovarian Syndrome is a common gynecological endocrinopathy characterized by chronic an ovulation and hyperandrogenism. The disorder is heterogeneous and is one of the most common treatable causes of infertility.
\end{abstract}

Objectives: To evaluate the clinical, biochemical and hormonal profile of patients with PCOS in Nepalese patients.

Methodology: A retrospective analysis of the clinical data and hormonal profile of patients with Polycystic Ovary Syndrome at Kathmandu Diabetes and Thyroid Center Pvt Ltd, Lalitpur, Nepal was undertaken.

Results: The mean age of 80 patients in this study group was $24 \pm 6$ years. Menstrual irregularity was the most common presenting problem occurring in $83 \%$ patients, followed by infertility (43\%) and hirsutism (33\%). Hyperandrogenism was demonstrated in $82 \%$. USG feature of Polycystic Ovarian Syndrome was apparent in $60 \%$ patients. The mean Body Mass Index was $27.4 \pm 5.1 \mathrm{~kg} / \mathrm{m}^{2}$. Diabetes, hypertension and hypothyroidism were noted among $2 \%, 4 \%$ and $30 \%$ of the patients respectively.

Conclusion: Our study concluded that menstrual irregularity was the most common presenting problem of Polycystic Ovarian Syndrome in our patient population. The condition is treatable and should be considered in patients presenting with infertility.

Keywords: amenorrhea, infertility, oligomenorrhea, polycystic ovarian syndrome, ovulatory, patients, syndrome, carcinoma, PCOS
Volume 4 Issue 2 - 2017

\author{
Ansu Mali Joshi, Priyadarshini Yonzon, \\ Sudarshan Tandukar \\ Kathmandu Diabetes and Thyroid Center, Nepal
}

Correspondence: Ansu Mali Joshi, DM Endocrinology, Consultant Endocrinologist, Kathmandu Diabetes and Thyroid Centre, Nepal, Email ansujoshi@yahoo.com

Received: August 22, 2016| Published: March 17, 2017

\section{Introduction}

Polycystic ovarian syndrome (PCOS) is the most common form of chronic an ovulation prevalent in reproductive aged women; it affects nearly $5-10 \%$ of women in child bearing ages. ${ }^{1}$ PCOS is a heterogeneous disorder with uncertain etiology and is characterized by androgen excess and polycystic ovaries. Ovulatory dysfunction is the most prominent clinical feature observed in PCOS. Hirsutism and acne may develop in some patients. The diagnosis of PCOS is made if two of the three criteria of androgen excess, ovulatory dysfunction, or polycystic ovaries are met. ${ }^{2}$ PCOS is associated with increased reproductive morbidity, including infertility, irregular menstruation and increased pregnancy loss. ${ }^{3}$ The etiology of PCOS is unknown but has significantly been associated with diabetes, risk for cardiovascular disease and endometrial carcinoma. ${ }^{4-6}$ This study has been conducted to assess the clinical and biochemical profile of Nepalese patients with PCOS.

\section{Methods}

A retrospective study was conducted between a period of March 2013 and October 2014, at Kathmandu Diabetes and Thyroid Center Pvt. Ltd., Lalitpur, Nepal, in which the data of patients diagnosed with PCOS were analyzed. A total of 80 patients were taken into consideration. Parameters such as age, BMI, hirsutism, ovulatory dysfunction, co-morbid conditions, concentration of serum androgen and USG of the patients were evaluated in the study. Hirsutism was evaluated using Ferriman Gallwey (FG) score. ${ }^{7}$ Polycystic ovaries were established when at least one of the ovaries demonstrates ovarian volume greater than $10 \mathrm{cc}$ or 12 or more follicles measuring 2-9 mm in diameter. ${ }^{2}$ A Serum testosterone level of $55 \mathrm{ng} / \mathrm{dl}$ was considered as a cut-off value for detection of hyperandrogenism. The data was entered and statistical analysis performed using Statistical Package for the Social Sciences (SPSS, version 21). Mean value and percentage were evaluated for PCOS.

\section{Results}

In this study group of 80 patients, the mean age at the time of presentation was $24 \pm 6$ years, with $59 \%$ of the patients in the age group of $20-29$ years. About $46 \%$ were married and $54 \%$ were single. Menstrual irregularity was observed in $83 \%$ of the patients, while the remaining patients had normal menses. Hirsutism was noted in $33 \%$, acne in $13 \%$ and infertility in $43 \%$ (Table 1). The mean Body Mass Index (BMI) was $27.4 \pm 5.1 \mathrm{~kg} / \mathrm{m}^{2}$ (Table 2). About $2 \%$ of the patients were diabetic while $4 \%$ of the patients had hypertension. Hypothyroidisim was observed in $30 \%$ of the patients. USG data on ovaries was available only for $69 \%$ patients. Among these, USG appearance of PCOS was observed in $60 \%$ patients. Total testosterone was elevated in $82 \%$ patients. Elevated level of prolactin was observed in $19 \%$. 
Table I Characteristics of Patients with PCOS

\begin{tabular}{lll}
\hline Character & Mean & Percentage \\
\hline Age & $24 \pm 6$ years & \\
Marital Status & \\
Married & $46 \%$ \\
Single & $54 \%$ \\
Hirsutism & $32.5 \%$ \\
Menstrual Irregularity & $83 \%$ \\
Infertility & $43 \%$. \\
Acne & $13 \%$ \\
BMI (kg/m $\left.{ }^{2}\right)$ & $27.4 \pm 5.1$ & \\
DM & & $2 \%$ \\
HTN & $4 \%$ \\
Hypothyroidism & $30 \%$ \\
Hyperprolactinemia & $19 \%$ \\
Elevated Total testosterone & \\
USG features of PCOS & \\
\hline
\end{tabular}

Table 2 Frequency of obesity in PCOS patients

\begin{tabular}{ll}
\hline BMI $\left(\mathbf{k g} / \mathbf{m}^{2}\right)$ & Frequency (\%) \\
\hline Underweight $(<18.5)$ & 7 \\
Normal (I8.5-24.9) & 24 \\
Overweight $(25-29.9)$ & 36 \\
Obese $(>30)$ & 33 \\
\hline
\end{tabular}

\section{Discussion}

Clinically diagnosing a woman as having PCOS implies an increased risk for infertility, dysfunctional bleeding, endometrial carcinoma, obesity, Type-II diabetes, hypertension, dyslipidemia, and possibly cardiovascular disease. ${ }^{8}$ In our study, the mean age of the patients with PCOS was 24 years indicating that it is a disease mainly of the young age. Menstrual irregularity was observed in $83 \%$ of our patients while study conducted by Vrunda Dhagat reported $65 \%$ in the same. ${ }^{9}$ The frequency of hirsutism was comparable to that noted in Chinese women (35\%), while acne was present only in $13 \%$ patients, as compared to $45 \%$ in the Chinese study group. ${ }^{10}$ Apparently, $57 \%$ of the married patients were fertile even though only $25 \%$ reported worldwide. ${ }^{11}$ Yet the overall prevalence of infertility could still be higher because most of the patients in this study were single (54\%).

In this study, obesity and overweight BMI groups were observed to be $33 \%$ and $36 \%$. This is quite different in comparison to $57 \%$ and $24 \%$ noted in a study in Libya ${ }^{12}$ and may indicate a lower BMI cut off values of $24 \mathrm{~kg} / \mathrm{m}^{2}$ for the risk of diabetes and other metabolic diseases in the South Asian population. Diabetes was observed only in $2 \%$ of the patients which is less than that reported in Asian women (17\%). ${ }^{13}$ This may be from the use of simple diagnostic tools rather than the use of glucose tolerance tests or HbA1C tests for diagnosis of the disease. Hypertension was reported only in about $4 \%$ patients, which is identical from the data observed in Libya (4\%). ${ }^{2}$ Hypothyroidism was observed in $30 \%$ of the patients. This is 10 times more than that that reported from Libya (3\%). ${ }^{12}$ Hyperprolactinemia was noted in $19 \%$ patients. This observation is similar to the $16.2 \%$ prevalence reported by another study. ${ }^{14}$

As this is a retrospective study, certain limitations have to be noted. The study failed to mention metabolic abnormality such as dyslipidemia which is an important indicator for possible cardiovascular diseases. However, this study, for the first time shows the various mode of clinical presentation and biochemical profile of patients with PCOS in Nepal.

\section{Conclusion}

PCOS not only is the most frequent cause of an ovulation, but is also associated with characteristic metabolic disturbances that may have important implications for the long term health. Our study, despite being of retrospective nature, concluded that menstrual irregularity and infertility are the major clinical presentations of PCOS. Yet, similar studies on a large scale would definitely be helpful to provide a more accurate picture of the disease.

\section{Acknowledgements}

We would like to thank our patient for being very cooperative during the entire work-up and for not missing her follow up appointments.

\section{Conflicts of interest}

The author declares there is no conflict of interest.

\section{References}

1. Dunaif A. Insulin Resistance and the Polycystic Ovary Syndrome: Mechanism and Implications for Pathogenesis. Endocr Rev. 1977;18(6):774 800 .

2. Fr DD, Tarlatzis R. Revised 2003 consensus on diagnostic criteria and long-term health risks related to polycystic ovary syndrome. Fertil Steril. 2004;81(1):19-25. 
3. Melmed S, Polonsky KS, Larsen PR, et al. Williams textbook of endocrinology $\left(12^{\text {th }}\right.$ edn.), Expert consult: Elsevier Health Sciences 1920.

4. Carmina E. Cardiovascular risk and events in polycystic ovary syndrome. Climacteric. 2009;12(Suppl 1):22-25.

5. Talbott E, Clerici A, Berga SL, et al. Adverse lipid and coronary heart disease risk profiles in young women with polycystic ovary syndrome: results of a case-control study. J Clin Epidemiol. 1998;51(5):415-422.

6. Legro RS, Kunselman AR, Dodson WC, et al. Prevalence and Predictors of Risk for Type 2 Diabetes Mellitus and Impaired Glucose Tolerance in Polycystic Ovary Syndrome: A Prospective, Controlled Study in 254 Affected Women. J Clin Endocrinol Metab. 199;84(1):165-169.

7. Ferriman D, Gallwey JD. Clinical assessment of body hair growth in women. J Clin Endocrinol Metab. 1961;21:1440-1447.

8. Azziz R, Marin C, Hoq L, et al. Health care-related economic burden of the polycystic ovary syndrome during the reproductive life span. J Clin Endocrinol Metab. 2005;90(8):4650-4658.
9. Dhagat V, Shah P, Thakar R, et al. Study of 100 cases of Infertility in Polycystic ovarian syndrome and its management outcome. Int J Med Sci Public Health. 2013;2(4):1041-1045.

10. Li L, Yang D, Chen X, et al. Clinical and metabolic features of polycystic ovary syndrome. Int J Gynecol Obstet. 2007;97(2):129-134.

11. Lakhani K, Seifalian A, Atiomo W, et al. Polycystic ovaries. British J Radiol. 2002;75(889):9-16.

12. Najem F, Elmehdawi R, Swalem A. Clinical and Biochemical Characteristics of Polycystic Ovary Syndrome in Benghazi-Libya; A Retrospective study. Libyan J Med. 2008;3(2):71-74.

13. Weerakiet S, Srisombut C, Bunnag P, et al. Prevalence of type 2 diabetes mellitus and impaired glucose tolerance in Asian women with polycystic ovary syndrome. Int J Gynecol Obstet. 2001;75(2):177-184.

14. Agbaht K, Yerlikaya H, Demir O. Hyperprolactinemia in polycystIc ovary syndrome. 2009. 